\title{
Diez conceptos claves para analizar el valor social de la educación física desde la voz de los estudiantes ${ }^{1}$
}

\author{
Javier Giles Girela² \\ Enrique Rivera García ${ }^{3}$ \\ Carmen Trigueros Cervantes ${ }^{4}$
}

Recibido 15 de noviembre 2018 - Aceptado 25 de noviembre 2018

\begin{abstract}
RESUMEN
¿Cuál es el verdadero objetivo de la educación física? ¿Qué debe aprender el alumnado? ¿Qué debemos hacer como futuros profesores de educación física? Muchos son los profesionales que tratan de dar respuesta a estas preguntas; sin embargo, pocos son los estudios que muestran reflexiones del alumnado acerca de estas cuestiones. Con el presente trabajo se pretende conocer qué piensan los alumnos acerca de la asignatura de Educación Física e intentar determinar, a partir de estas reflexiones, un objetivo que defina a la misma tal y como se merece. En este estudio, de carácter cualitativo, participaron I4 estudiantes de tercer año de Educación Media durante el periodo 2016/2017.
\end{abstract}

Palabras clave: valor, alumnado, mesa cuadrada, educación, "physical literacy".

Ten key concepts to analyze the social value of physical education from the voice of students

\begin{abstract}
What is the true objective of Physical Education? What should students learn? What should we do as future teachers of physical education? There are many professionals who try to answer all these questions, however, there are few studies showing students' reflections about these questions. This work is aimed at knowing what students think about the subject of Physical Education and, from these reflections, determining their defining objectives. This qualitative study included I4 third-grade students of Secondary Education during the 2016/2017 academic year.
\end{abstract}

Keywords: Value, students, square table, education, "physical literacy."

1 Proyecto patrocinado por el grupo de investigación "Educación Física y transformación social", de la Universidad de Granada (España).

2 Postgraduado en Educación, Universidad de Granada, España. E-mail: javiggr@hotmail.com

Doctor en Educación Física, Universidad de Granada, España. E-maili erivera@ugr.es

4 Doctora en Educación Física, Universidad de Granada, España. E-mail: ctriguer@ugr.es 


\section{Introducción}

A pesar de estar frente a una asignatura consolidada en el currículo escolar de la enseñanza no universitaria, en los últimos años la Educación Física (EF) no ha sido capaz de encontrar su razón de ser. Según Smith y Parr (2007), saber y conocer su papel y objetivo en los centros es desde hace mucho tiempo un acontecimiento complicado. Además, muchos de los investigadores que tratan de establecer un objetivo definitivo para esta asignatura están aún en la búsqueda de éste (Alderson \& Crutchley, I990; Bailey \& Kirk, 2009; Kirk, 1992, 2013; Whitehead, 2010a). En este sentido, la EF no ha sabido mostrar identidad propia, si es que la tiene, sino que ha actuado como un espejo al servicio de otros intereses (Martínez Álvarez, 2000).

La identificación de una justificación clara para la EF está relacionada con un apoyo político y una seguridad curricular, dependiendo su futuro de la capacidad de establecer un consenso unificado en cuanto a su propósito (Sprake \& Walker, 20I5). La asignatura se ha caracterizado por un juego político, a partir del cual puede ser expulsada o introducida en el currículo dependiendo de los intereses particulares de los gobiernos (Johnrose \& Maher, 2010). Actualmente, la EF en primaria se ve trastocada con la LOMCE. Suponemos que, si ya ocurre en este nivel educativo, ocurrirá también en secundaria. Queda en manos del equipo directivo el número de horas que se puede dedicar a esta materia y se considera necesario reducir sus horas para "equilibrar" el resto de las asignaturas. Sin embargo, la LOMCE también afirma como fundamental acabar con la obesidad infantil y el sedentarismo, que ahora mismo se han identificado como dos de los problemas que afectan a nuestra sociedad y en un futuro será aún mayor, aunque, como hemos señalado anteriormente, esto no se ve reflejado en la reducción de horas que esta padece. PérezLópez (20I I) afirma que la EF cuenta con argumentos suficientes para justificar su presencia en el curriculum actual, sin necesidad de venderse mediante una imagen "atractiva" pero vacía, quedando presionada intentando cumplir con las expectativas de la sociedad acerca de ella. Deberíamos aprender de países cercanos, como Gran Bretaña, en el que es tratada como materia obligatoria, junto con 
inglés, matemáticas y ciencias, a lo largo de las cuatro etapas clave que se establecen desde el Departamento de Educación (2013).

Siguiendo con esta idea, esta asignatura tiene gran incapacidad para lograr imponerse socialmente como una disciplina escolar relevante. Hardman y Marshall (2000), en sus estudios, recogen referencias de la marginación de la asignatura, de su estatus inferior y de la imagen de baja estima que existe al respecto por parte de grupos significativos en el ámbito escolar. Para invertir esa imagen, Pérez-López (20I I) señala que los profesores de EF debemos estar plenamente convencidos de sus posibilidades y vivir como una auténtica vocación nuestra labor educativa. Además, este mismo autor añade la necesidad de ser autocríticos con la profesión, si verdaderamente pretendemos dar un salto de calidad que ayude a mejorarla y dignificarla. Los profesionales de este ámbito debemos identificar y articular una visión clara y el propósito para la EF (Whitehead, 2000). Muchas son las características positivas que definen la asignatura; entre sus señas de identidad destacan valores como el compromiso, la solidaridad, el trabajo en equipo o el afán de superación, que difícilmente se encuentran tan arraigados en el profesorado del resto de asignaturas (Pérez-López, 20I4). En las manos del docente de EF está superar la situación de "marginación" que sufre, destacando aquí lo que dice Rivera (200I): "sin él, toda posibilidad de cambio no dejará de ser más que una excelente declaración de buenas intenciones” (p. 625).

En la búsqueda de ese objetivo y sentido de la EF, Whitehead (2013), propone un nuevo concepto: physical literacy, que se describe como la capacidad de tener la motivación, confianza, capacidad física, conocimiento y comprensión de valorar y participar en la actividad física durante todo el ciclo vital. Añade que esta propuesta podría ser el resultado para propiciar en los estudiantes las capacidades y disposiciones con los cimientos necesarios sobre los cuales construir un compromiso de por vida y de disfrute de la actividad física. El estudiante que se desarrolla en este sentido, tiene la capacidad de moverse con confianza y competencia en una gran variedad de actividades y ambientes diferentes, favorecedores del desarrollo integral de la persona. La misión de esta propuesta es la de capacitar a las personas de todos los niveles y edades para 
la incorporación de la actividad física en su vida, teniendo aquí una oportunidad para encontrar el propósito primordial de nuestra asignatura y presentar una justificación para el plan de estudios (Whitehead, 2010). Como señalan Sprake y Walker (2015), hay que tener presente que el alumnado entrará en nuestra aula en su propio viaje de physical literacy, por lo que el desarrollo integral de cada persona será individual y personalizado, aunque siempre en relación con otros. No se trataría tanto de educar físicamente al alumnado, aprovechando las peculiaridades y oportunidades que brinda el área, sino de contribuir a la construcción de la personalidad de los alumnos y a su formación integral, lo que constituye la principal finalidad de todo acto educativo (Pérez-López, 201 I). Partir del conocimiento del territorio, de los estudiantes que lo habitan, debe ser el punto de partida de toda planificación. Serán estos condicionantes los que mediatizarán las teorías implícitas del docente (Pérez-López, 20I5).

¿Qué debemos hacer como futuros profesores de EF si optamos por esta metodología? Respecto de esta pregunta, Whitehead y Almond (2013), proponen que los profesores deben considerar varias experiencias del alumnado, experiencias gratificantes y agradables: promover la autoestima y la autoconfianza, que todos progresen y tengan éxito, enseñar al alumnado a tomar decisiones sobre lo que están haciendo, enseñarles a comprender el valor de la actividad física para los beneficios de la salud y propiciar experiencias que los estimulen a participar en la actividad física por sí mismos. Añadir, además, tres pilares básicos para posibilitar el éxito: talento, compromiso e ilusión (Pérez-López, 20I4). Un aspecto que nos gustaría destacar aquí es la forma de obtener el éxito en la posible evaluación llevada a cabo en la asignatura.

En este sentido, Rimmer (2013), señala que el éxito de los alumnos en la EF debe entenderse en términos de su "experiencia de éxito", y se mide mediante la interpretación de lo que está "escrito en las caras de nuestros estudiantes”. Whitehead (2010), señala que los estudiantes deben ser juzgados solo en relación con sus propias actuaciones previas, en lugar de ser comparados con otros o con criterios de éxito predeterminados. La educación física debe sorprender, ser transformadora e infundir proactividad (Pérez-López, 2015). 
Según estos antecedentes, podemos llegar a conocer lo que piensan muchos de los profesionales de nuestro ámbito; sin embargo, no se conocen estudios que muestren lo que el alumnado piensa sobre nuestra asignatura. Por tanto, ahora más que nunca, la participación en este tipo de debates es una responsabilidad esencial de los profesionales de la EF para llegar así a conclusiones significativas y tangibles (Sprake \& Walker, 2015).

El principal objetivo de este estudio ha sido conocer qué piensa el alumnado acerca de nuestra asignatura y, a partir de sus reflexiones, intentar determinar los ejes que definan a nuestra intervención didáctica.

\section{Metodología}

Metodológicamente hemos optado por un estudio de corte fenomenológico, en el que las teorías de los participantes serán el eje en torno al que gire todo el análisis. Entendemos que el objetivo marcado inicialmente no deja de ser meramente orientador, dejando que sean las propias preguntas de los investigadores y las teorías implícitas de los participantes las que marquen el rumbo de la investigación.

El diseño de la investigación es de carácter evaluativo y ha seguido las pautas de la investigación cualitativa, marcadas por Glaser y Strauss (I967) desde la Teoría Fundamentada, centrada en tres claves: detección de conceptos relevantes desde la inducción, saturación de los datos y construcción de teorías sustantivas (Cuñat 2007; Soneira 2006; Strauss \& Corbin, 2002).

\section{Participantes}

Los participantes del estudio fueron I4 estudiantes ( 6 alumnos y 8 alumnas) de $I^{\circ}$ de Bachillerato ( $4^{\circ}$ de Enseñanza Media). Su implicación en esta propuesta metodológica fue totalmente voluntaria. Para la selección de los participantes se tuvo en cuenta las reflexiones previas enviadas por una parte del alumnado de la clase tras leer el texto matriz acerca de ¿qué es para ti la educación 
física?, siendo este un texto base que expresó las teorías implícitas de las que se partiría.

\section{Procedimiento}

El diseño del estudio se ha realizado en dos fases: una de reflexión previa, a partir de un texto matriz, y realización de la técnica de "mesa cuadrada" (Caetano, 2013), en la que se llevó a cabo una reflexión en común.

En la primera fase el alumnado participante recibió un texto matriz acerca del que tenían que realizar una reflexión y enviarla por correo para ser analizada. La reflexión que debían hacer era respecto de: ¿qué es para ti la educación física? Una vez realizada la reflexión y enviada por correo, se llevó a cabo una selección de I4 participantes para la segunda fase. Los participantes, antes de realizar la mesa cuadrada, recibían en su correo las $\mathrm{I} 4$ reflexiones resumidas realizadas por los compañeros de forma anónima, para leerlas antes del día de la mesa cuadrada.

En la segunda fase se llevó a cabo la mesa cuadrada. Respecto de la distribución de los participantes, estos se posicionaron en torno a un espacio cuadrado. Dicha metodología fue automoderada por sus participantes, los cuales tenían el turno de palabra siempre abierto. Además, fueron ayudados por un moderador externo, que solo intervenía para organizar el turno de palabra, presentar la mesa y reconducir el diálogo cuando era preciso. Una segunda persona colaboró para la toma de notas y facilitar posteriormente la transcripción del diálogo generado. La duración de la mesa cuadrada fue de aproximadamente una hora y cincuenta minutos.

Esta segunda fase (mesa cuadrada) se dividió en tres momentos: a) cada participante contó al resto su reflexión acerca de la EF. En esta estapa podían ayudarse de las reflexiones enviadas previamente o de algunas notas que hubieran tomado para traer a la mesa; b) en este segundo momento, denominado periodo de debate, tras haber escuchado las reflexiones de los demás participantes, se pasa a la exposición argumentada de sus propuestas, generándose el debate propiamente dicho. Por último, c) se pasa al momento 3, en el que 
se construyen las conclusiones que deben ser consensuadas entre todos los participantes.

\section{Estrategias para el análisis de la información producida}

Siguiendo a Caetano (2013), la mesa cuadrada es una técnica que se basa en las orientaciones marcadas por Civiliter. Utiliza estrategias del grupo de discusión, está inspirada en la utilizada por el Pacto Mundial de Naciones Unidas en sus reuniones de progreso y posibilita la participación de todos los actores que intervienen sobre una realidad, enriqueciendo el trabajo de introspección y prospección sobre dicha temática. No es recomendable la participación de más de 20 personas, siendo el segmento I2-I6 el número ideal.

Todo el proceso de la mesa cuadrada fue grabado en audio y posteriormente transcrito con el software NVivo para facilitar su análisis.

El proceso de análisis ha partido de una frecuencia de palabras que, de forma progresiva, se ha ido concretando hasta llegar a los diez conceptos con mayor presencia en los discursos de los participantes. Este análisis se ha centrado en el diálogo generado en la mesa cuadrada.

Posteriormente se procedió a la categorización y codificación de estos términos, transformándose en categorías centrales, que han sido analizadas y saturadas desde la creación de las subcategorías anidadas en ellas. Las diez categorías centrales se concretaron en: vida; salud; docente; personas; importancia; tiempo de dedicación; felicidad; educación física; cambios y adaptaciones.

A partir de esta primera aproximación inductiva al discurso, se pasó a un segundo análisis de carácter deductivo, que nos ofreció las claves de las relaciones producidas entre las categorías centrales. Además, se buscó la significatividad de los discursos desde la construcción de matrices singulares, en las que se quería detectar la presencia o ausencia de las categorías centrales en el discurso particular de cada participante. 


\section{Análisis e interpretación de resultados}

Desde la marca de nube conceptual generada a partir de la frecuencia de palabras realizada como primer paso del proceso de análisis, se puede constatar cómo los diez conceptos que posteriormente construyen las categorías centrales están presentes con fuerza en ella. Lógicamente aparecen todos los conceptos del discurso, aunque muchos de ellos fueron posteriormente desechados por no ser considerados relevantes en esta investigación. Desde esta primera aproximación cartográfica, podemos concretar aún más gracias a la frecuencia generada y decir que los conceptos "educación física" y "tiempo de dedicación” (horas) están presentes en todos los discursos de los participantes. "Personas", "profesor", "importancia”, "felicidad" y "vida” tienen una significativa presencia. Una presencia discreta tienen los conceptos de "salud" y "cambios". Con poca significatividad se encuentra "adaptaciones".

Imagen I. Marca de nube conceptos Nvivo.

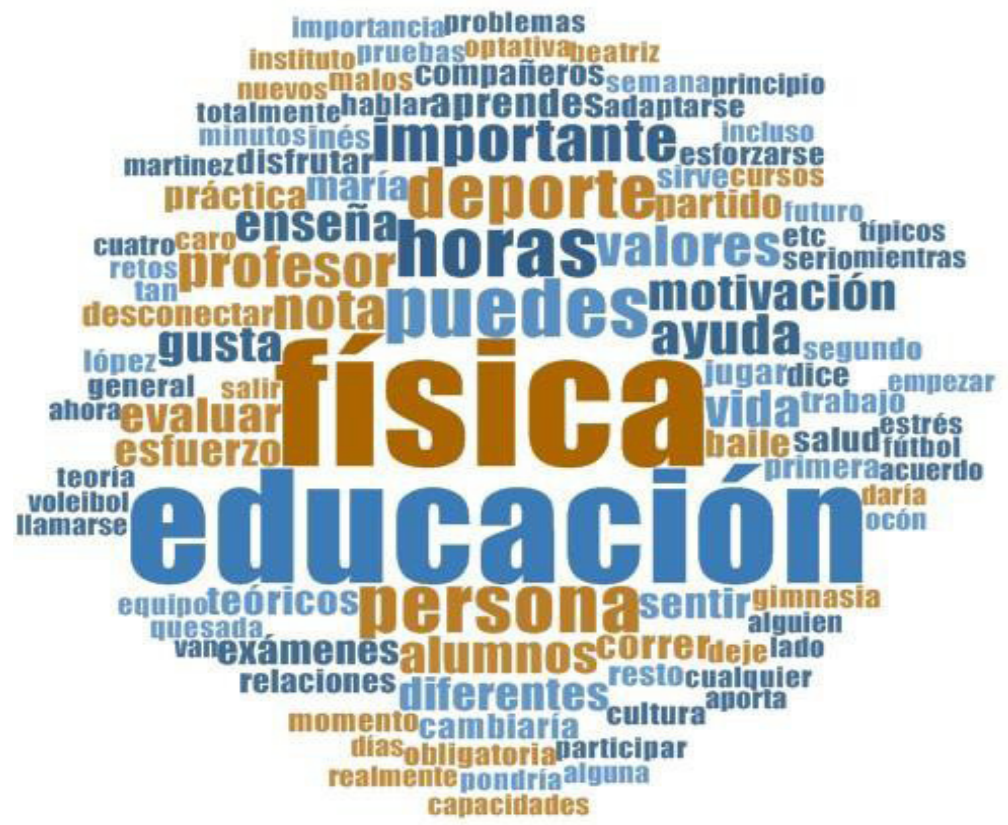

Fuente: elaboración propia. 
¿Qué piensan de la EF en general? En primer lugar, podemos observar que esta idea se revela como un tema clave para todos los participantes y que su consideración como asignatura sigue siendo de carácter "especial”. Sparker y Walker (20I5) ya nos adelantan esta idea cuando hablan del escaso valor curricular de la EF. Manuel ${ }^{\mathrm{I}}$ piensa que "la educación física pues, es una de las asignaturas más importantes que podemos tener, aunque no parezca tanto como las demás”. Idea que corroboran Rocío y Julia, respectivamente, cuando comentan que la forma de aprendizaje difiere del resto de asignaturas: "Yo creo que la educación física es como otra forma de aprender, porque no es lo típico de simplemente sentarte en una silla”. Por su parte, Rocío entiende que "la educación física es una asignatura totalmente diferente a las demás”. Sara pone especial énfasis en el poco valor que recibe respecto de las demás asignaturas: "La educación física no se toma demasiado en serio, porque a veces las demás asignaturas le achacan que no nos aporta lo mismo que ellas, muchas veces no se valora lo que nos puede aportar”. Esta línea la defienden Hardman y Marshall (2000), entre otros autores.

¿Qué opinan acerca del tiempo que se dedica a la EF? Un tema relevante para todos los participantes. La mayoría remarca la necesidad de aumentar el número de horas actuales. Julio nos dice que: "Sin embargo, sí que hay cosas que habría que revisar, como es el número de horas que hay". El tiempo durante las clases de EF pasa muy rápidamente, así lo señala Rosa diciendo: "El tiempo vuela y casi cuando quieres acordar la hora ha terminado, no se te ha hecho pesada esta clase, no como cualquier clase de historia o filosofía que te puede resultar algo más pesada sin poder evitar quitar un ojo a las agujas del reloj”.

Además, Rosa señala que sería positivo tener un mayor número de horas de EF, haciendo referencia a la desconexión que le produce del resto de asignaturas: "desde mi punto de vista, creo que esta debe ser impartida un mayor número de horas semanales, ya que es una clase que permite al alumno desconectar de todas las clases anteriores”. Este aumento del número de horas, poco factible en el

Los nombres que aparecen son ficticios, para salvaguardar la identidad del hablante. 
actual modelo curricular, ya lo defiende Pérez-López (20I I); pero quizás la experiencia nos dice que, antes de solicitar más tiempo, hay que definir con claridad un nuevo modelo de EF, como ya sostenían Kirk (2013) y Whitehead (2010).

Otra de las preguntas claves es si realmente la EF es capaz de enriquecer la carga valórica del estudiantado. En este punto, es cierto que la mayoría de los discursos se orientan hacia una clara mejora de los aspectos sociales, tal y como explicita Rocío, cuando afirma que la asignatura "mejora los aspectos sociales de las personas, porque trabajas en equipo y tienes que poder comunicarte con tu equipo para poder realizar deporte"; o como apunta Sergio en su discurso, destacando la virtud de la EF para aportar afán de superación y capacidad de afrontar los problemas correctamente. Estas afirmaciones son compartidas por autores como Pérez-López (2014), pero nos atrevemos a cuestionarlas al reflexionar si estas mejoras llegan a todo el alumnado, o solo a la parte privilegiada con la suficiente competencia motriz que lograr obtener experiencias de éxito en las clases de EF.

¿Por qué la EF es importante para el alumnado? Rocío señala la importancia de las relaciones y la comunicación que en esta clase se $\mathrm{da}$, indicando la similitud que tiene con la realidad: "la asignatura que más nos acerca a la realidad, ya que aprendes cosas básicas y, lo más importante, aprendes a relacionarte y a comunicarte con los demás, que es lo más importante en la vida”. Julia destaca como importante la diversión que les aporta la clase respecto de las demás asignaturas: "la Educación Física es una asignatura tremendamente importante, pero bastaría simplemente una pequeña y sencilla razón: es divertida. ¿Se puede decir eso de todas las grandes y respetadas asignaturas?”.

La salud es un aspecto importante que señalan en más de una ocasión: "desde una temprana edad te enseñan el valor del ejercicio físico, ya no el deporte sino el ejercicio físico en general; entonces, aparte de que te ayuda a socializar o te ayuda también con los problemas de salud o los valores de equipo" (Javier).

¿Qué opinan acerca de la figura del profesor? Javier remarca la importancia de la motivación que este debería tener en sus clases: 
"lo importante es la motivación no solo del profesorado sino también de los alumnos". Carmen está de acuerdo con Javier y añade que "si el profesor te motiva, tú te sientes motivado". Otro de los aspectos al que hacen referencia los participantes es el de los métodos que el profesorado pone en práctica en sus clases. En este sentido Rocío defiende las metodologías innovadoras frente a las tradicionales: "que no repita siempre las típicas actividades y los típicos deportes de todos los años, que hagan cosas nuevas”. Por último, Víctor señala como solución "apostar por la motivación personal del profesorado y los alumnos [como] la única forma posible de salir hacia adelante".

¿Qué enseñanzas aporta la EF en la vida del alumnado? Los participantes indican aportaciones en valores que utilizarán en sus vidas, Juan es tajante en este tema: "lo que la educación física te enseña es como una forma de vida, es enseñarte una serie de valores que luego tú no solo los vas a practicar en la educación física, sino también en toda tu vida”. Como vemos aquí, Juan hace referencia a valores que la EF aporta para su vida, en este sentido, Javier añade un objetivo de mejora de la calidad de la participación humana a ese aporte de valores que se convierte en necesidad social:

$[\ldots]$ la educación física aporta la mejora de capacidades diversas, como la afectiva o la cognitiva, que tienen como objetivo la mejora de la calidad de la participación humana en los distintos ámbitos de la vida, como son el familiar, social y productivo. Es justo decir que se trata de una necesidad, no solo individual, sino también social.

Juan destaca en concreto unos valores que se adquieren en las clases de EF sin darse cuenta de esa adquisición: "es una forma de fomentar otros valores cruciales en la vida, como son el esfuerzo, el compañerismo, la empatía... al mismo tiempo que se realiza una actividad saludable. Lo mejor de todo es que esto se adquiere sin darte cuenta”. Además, Raquel señala que la EF aporta a la vida una herramienta para evitar un estilo de vida sedentario, señalando: "con el estrés de los estudios daremos de lado al deporte cayendo en una vida sedentaria e infeliz, mientras que la Educación Física es la herramienta educativa más eficaz contra esto si se plantea bien”. 
Como podemos observar a través de los discursos de los estudiantes, la EF es una asignatura valorada por los alumnos, por su aporte a la construcción de los valores personales. Este punto lo veíamos anteriormente en Pérez-López (2014). Pero, al mismo tiempo, el testimonio de los alumnos deja en evidencia una asignatura que queda indefinida y carente de otros objetivos que vayan en la idea de construir saberes y quehaceres en nuestro alumnado (Smith y Parr, 2007; Bailey \& Kirk, 2009; Whitehead, 2013).

Desde el análisis anterior, podemos decir que la EF es un generador de felicidad. Cabe preguntarse si esta afirmación es válida para todo el alumnado o también tenemos una parte del mismo que no es tan feliz en las clases. Quedándonos con los discursos del alumnado, debemos destacar que se sienten mayoritariamente felices en clase y que esta sensación provoca en ellos adherencia a la práctica de ejercicio porque creen "que simplemente por el hecho de cuando haces educación física, la sensación de bienestar y felicidad que te aporta va a hacer que luego, fuera del instituto, quieras realizarlo también” (Julia). Utilizan esta relación para definir a la asignatura "como una expresión pura de vitalidad y felicidad a través del mismo ejercicio físico" (Javier). Estamos ante una clase con un "clima mucho más distendido, divertido y alegre, y el buen hacer del profesor hace que, lo hagas como lo hagas, te sientas bien” (Julia).

¿Quién debe adaptarse? ¿La asignatura a los estudiantes o ellos a la asignatura? Ante esta disonancia, coinciden en todas sus intervenciones que "la educación física tiene que adaptarse a los alumnos no los alumnos a la educación física [...] Los profesores deberían proponer algo para que los alumnos pudiesen elegir qué hacer y qué no hacer" (Martín). Dentro de esta misma idea, Carmen centra el foco en la evaluación, especialmente en los tests de valoración de las capacidades físicas, dejando claro que "los baremos se deberían de adaptar al tipo de personas que van a realizar esa actividad física”.

¿Cuáles son los cambios que proponen para adaptar la EF a sus necesidades? Un aspecto que señalan importante y que debería tenerse en cuenta es la evaluación de la asignatura. Así, Juan remarca lo dicho 
por Carmen, mientras que Rocío pone el foco en la desmotivación que provoca en algunas ocasiones el poner calificación:

un simple número lo puede cambiar todo y, en vez de tomarlo como algo positivo, lo vemos, si no tenemos quizá la gran destreza en los deportes que tienen otros, como algo todo lo contrario y produce en nosotros una desmotivación, y se nos quitan las ganas de seguir practicando deporte.

Tratando de sintetizar el análisis interpretativo realizado, queda en evidencia que el alumnado ve a la EF distinta del resto de asignaturas. El alumnado es consciente de esa imagen desvirtuada que señala Rubia (2013). En nuestras manos, como profesionales del ámbito de EF, está el poder de cambiar esa imagen, y de nuestros actos dependerá el elevar el estatus y el valor real de nuestra asignatura. Como hemos visto en los resultados analizados, la mayoría de los participantes indican gran cantidad de aspectos positivos, remarcando la importancia de la asignatura en su aprendizaje.

Coinciden mayoritariamente con Pérez-López (2015), en que la asignatura propicia que el alumnado viva una experiencia de aprendizaje que le supone un enriquecimiento para sus vidas y les hace crecer como personas, mejorando facetas imprescindibles en su día a día, como por ejemplo el trabajo en equipo y la comunicación. Confirmando así algunas de las señas de identidad de nuestra asignatura, como el compromiso, la solidaridad, el trabajo en equipo y el afán de superación que Pérez-López (20I4) destacaba anteriormente.

Respecto del papel del profesorado de la asignatura, la mayoría de los participantes coinciden en que se debería poner en práctica nuevas metodologías: dejar a un lado lo tradicional e innovar en las actividades, confirmando así lo que dice Rivera, (200I), acerca de que en nuestras manos está el poder superar la situación de "marginación" que sufrimos, señalando que, sin nosotros, toda posibilidad de cambio no dejará de ser más que una excelente declaración de buenas intenciones.

La relación de la salud con la EF es un tema remarcado por los participantes. La mayoría de estos coinciden en la importancia de la 
asignatura para ayudar a mantener la y evitar el sedentarismo, por lo que están de acuerdo con el apartado de la LOMCE que defiende este concepto.

Además, la mayor parte de ellos coincide en que EF es la asignatura de la felicidad y todos disfrutan de las actividades que en ella se realizan, coincidiendo así con Pérez-López (20I4) cuando señala el deber de convertir nuestra asignatura en la asignatura de la felicidad, una inigualable y enriquecedora experiencia de aprendizaje para toda la vida.

Con relación a las adaptaciones propuestas por los participantes, una parte de ellos coincide con Rimmer (20I3) y Whitehead (2010) en que la asignatura debe adaptarse a las características de todos los alumnos y no poner en práctica una evaluación basada en criterios de calificación predeterminados. En este mismo sentido, y haciendo referencia a los cambios que proponen, la mayoría de ellos coincide en que se debe revisar la forma de evaluación en determinadas ocasiones, remarcando aquí una de las conclusiones de Rubia (2013), en la que señala que muchas materias prácticas se transforman en asignatura "de libro y de examen" para reforzar su valor académico y huir de su imagen de "María", haciendo prevalecer el verdadero valor que tiene nuestra asignatura y dejando de lado esta transformación.

\section{Conclusiones y limitaciones del trabajo}

Las conclusiones más significativas a las que podemos llegar sobre las opiniones vertidas por parte de los estudiantes las podemos concretar en la siguiente tríada.

Existe una clara coincidencia en relación con el necesario aumento del tiempo curricular dedicado al área, aunque desde una perspectiva utilitaria y poco enriquecedora para la adquisición de conocimientos no centrados en el hacer. Desde una posición crítica, estamos de acuerdo en que, antes de aumentar el tiempo dedicado a EF, debiéramos analizar el para qué de la asignatura y definir con claridad sus objetivos, para así pensar un cambio, es necesario una reflexión previa.El alumnado percibe la asignatura con una fuerte carga valórica, pero escasamente se refiere a ella como un tiempo de 
aprendizaje para profundizar en aquellos contenidos que refuercen un claro conocimiento del qué estoy haciendo y el para qué se está realizando. Aplauden la motricidad acrítica y feliz, que lo único que aporta es el momento del juego y la compensación de tantas horas en el aula convencional.

Cuando hablan de cambios y adaptaciones se quedan en la superficialidad, entrando tan solo en contenidos superficiales que, además, poco o nada tienen que ver con los estándares de aprendizaje marcados en el currículo. Buscan una asignatura que se adapte a sus necesidades y que les aporte la felicidad efímera del momento. No se encuentra en sus discursos el mínimo atisbo de crítica al hacer del docente o a los objetivos de la materia. Esto quizás justifique el inmovilismo de una asignatura que, prácticamente, salvo excepciones, mantiene las mismas formas de hacer que en el pasado reciente.

\section{Referencias bibliográficas}

Alderson, J. y Crutchley, D. (1990). Physical education and the national curriculum, in Armstrong, N. (ed.), New Directions in Physical Education 1 (pp. 37-62). Illinois: Human Kinetics.

Bailey, R. y Kirk, D. (2009). The Routledge Pbysical Education Reader. Oxon: Routledge.

Caetano, B. (2013). Participación y diversidad. Avanzando en la participación de las personas inmigrantes en la sociedad civil de Andalucía. Sevilla: Dirección General de Coordinación de Políticas Migratorias. Consejería de Justicia e Interior. Junta de Andalucía Coedita; Fundación Civilite.

Cuñat, R. J. (2007). Aplicación de la teoría fundamentada (grounded theory) al estudio del proceso de creación de empresas. Decisiones basadas en el conocimiento y en el papel social de la empresa: XX Congreso anual de AEDEM. Vol.2, pp. 44. Recuperado de http://dialnet.unirioja.es/servlet/articulo?codigo $=2499458$.

Department for Education (2013a) National curriculum review launcbed. Recuperado de: http://www.education.gov.uk/inthenews/inthenews/a0073I49/ national-curriculum-review-launched (accessed 3 April 2013).

Glaser, B. y Strauss, A. (1967). The Discovery of Grounded Theory. Strategies for Qualitative Research. Chicago: Aldine Publishing Company.

Hardman, K. y Marshall, J. (2000). World-Wide Survey of the State and Status of School Pbysical Education. Manchester: University of Manchester. 
Johnrose, W. y Maher, A., (2010). National curriculum physical education: Health lifestyles and lifelong participation in physical activity. Journal of Qualitative Research in Sports Studies, 4(I), I5-22.

Kirk, D. (1992). Defining Pbysical Education: The Social Construction of a School Subject in Postwar Britain. London: The Falmer Press.

Kirk, D. (2013). Educational value and models-based practice in physical education. Educational Pbilosopby and Theory, 45(9), 973-986.

Martínez Álvarez, L. (2000). A vueltas con la historia: una mirada a la Educación Física escolar del s. XX. Revista de Educación, Nºxtraordinario, 83-II2.

Pérez-López, I. J. (20I I). Una cenicienta llamada María. Educación Física y deporte escolar. IX Congreso Internacional sobre la enseñanza de la Educación Física y el Deporte Escolar.

Pérez-López, I. J. (2014). ¡Este partido... Lo vamos a ganar! EmásF, Revista Digital de Educación Física, 5(30).

Pérez-López, I. J. (2015). Desaprende a programar en 1000 palabras (basado en hechos reales). EDITORIAL Habilidad Motriz, 44.

Rimmer, V. (2013). What is success in physical education and how can this best be achieved?, in: Capel, S. and Whitehead, M. (eds.), Debates in Physical Education (pp. 89-106). Oxon: Routledge.

Rivera, E. (200I). Vamos a contar mentiras. Mejorar la práctica de la Educación Física desde el diseño o viceversa, en Díaz, A. y Segarra, E. (coords.), Actas del II Congreso Internacional de Educación Física y Diversidad (pp. 617633). Murcia: Dirección General de Centros, Ordenación e Inspección Educativa.

Rubia, A. F. (2013). La LOMCE, una ley que apuesta por las desigualdades sociales. Fórum Aragón, (7).

Smith, A. y Parr, M. (2007). Young people's views on the nature and purposes of physical education: A sociological analysis. Sport, Education and Society, 12(I), 37-58.

Sprake, A. y Walker, S. (20I5). "Blurred lines": The duty of physical education to establish a unified rationale. European Pbysical Education Review, I-I3.

Strauss, A. y Corbin, J. (2002). Bases de la investigación cualitativa. Técnicas y procedimientos para desarrollar la teoría fundamentada. Colombia: Editorial Universidad de Antioquia.

Whitehead, M. y Almond, L. (2013). Creating learning experiences to foster physical literacy. Physical Education Matters, 8(I), 24-27. 
Whitehead, M. (2000). Aims as an issue for physical education, in Capel, S. \& Piotrowski, S. (eds.), Issues in Pbysical Education (pp. 7-2I). London: Routledge/Falmer.

Whitehead, M. (2010). Aims of PE, in Capel, S. y Whitehead, M. (eds.), Learning to Teach Pbysical Education in the Secondary School (3rd edn) (pp.I323). London: Routledge.

Whitehead, M. (2013). What is physical literacy and how does it impact on physical education?, in: Capel, S. y Whitehead, M. (eds.), Debates in Physical Education (pp. 37-54). Oxon: Routledge. 